tan unos $10 \mathrm{~cm}^{3}$ de disolución y se añade gelatina de repostería; cuando ésta se ha disuelto, se vierte en la tapa de una caja Petri, formando una capa de $1-2$ mm y se deja cuajar.

- Construir, del modo descrito en la figura, un sistema que permita hacer girar el recipiente que contiene la disolución junto con el analizador, respecto del polarizador y el goniometro, que permanecen fijos.
- Con el montaje descrito, se puede proceder a verificar experimentalmente la ley de Biot, variando la concentración y el espesor de líquido y midiendo en cada caso el ángulo que hay que girar la vasija y el analizador con respecto al polarizador, para que desaparezca completamente la iluminación proyectada a través de la disolución. También se puede determinar la rotación específica de la sacarosa 1 otras sustancias opticamente activas.

\section{Bibliografía}

Allinger et al. Quimica Orgánica. (Reverté).

Llorens, J.A. Demostraciones experimentales de Química con retroproyector. (CEP Gandía. Consellería de Educación de la Generalitat Valenciana).

\title{
LINEAS DE TRABAJO
}

\section{MEDICIÓN DE INTERESES EN FÍSICA $Y$ TECNOLOGÍA EN CHILE}

\section{María Calderón Brito.}

Departamento de Fisica. Facultad de Ciencias. Universidad de Concepción Chile.

Teniendo en cuenta que la mayoría de los alumnos chilenos considera la asignatura de Ffsica, difícil, poco atractiva, abstracta y desconectada de la vida real, y dado el descontento de los profesores con los actuales programas, es un hecho que deberfa haber en un futuro cambios en los actuales programas de Física. En el pasado se han intentado diferentes enfoques curriculares en Física, sin tener en cuenta algo muy importante como es la información acerca de los intereses de los alımnos respecto de Física y cómo cambian éstos en los diferentes niveles de escolaridad; por ello quizá todas estas innovaciones no han producido mejoras substanciales en el proceso y el espectro de dificultades subsiste a través del tiempo.

Teniendo en cuenta que los estudios realizados por diferentes investigadores educacionales (Claparede, Super, Dewey,
Fryer, Gadner, Van Aalst, etc.) han mostrado a través del tiempo que los intereses constituyen una variable muy importante en el aprendizaje y que por ende, para realizar cualquier modificación curricular deberían tenerse muy en cuenta; un grupo de profesores de la Universidad de Concepción de la Facultades de Educación y de Ciencias hemos organizado un Proyecto de Investigación que tiende a detectar los intereses de los alumnos chilenos respecto a Física y Tecnología. Se espera que a partir de los resultados obtenidos de este trabajo, puedan hacerse las modificaciones curriculares en Física en el futuro.

Para llevar a cabo la detección del espectro de intereses de los alumnos chilenos, se utiliza una versión española de un cuestionario creado por un grupo de investigadores del Institut fur die Padagogik de Naturwissenschaften (I.P.N.) en Kiel, Alemania Federal. De dicho cuestionario se hizo una adaptación y un estudio de validez y confiabilidad en la población estudiantil chilena.

\section{Fun damentación y estructura del cues-} tionario

La serie de trabajos preliminares que sirvieron de base para la elaboración de este cuestionario de intereses, llevo a los investigadores de la Universidad de Kiel a considerar que el interés de cada individuo en relación a Física, depende del Topico o tema considerado, del contexto particular con el cual dicho tópico está asociado y del tipo de actividad o accion que oriente su aprendizaje.

Para la elaboración del cuestionario se consideraron 8 tópicos, 5 contextos y 4 niveles de acción, como se muestra en el cuadro siguiente.

\begin{tabular}{|c|c|c|}
\hline 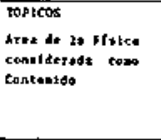 & 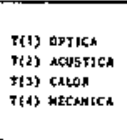 & 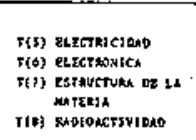 \\
\hline 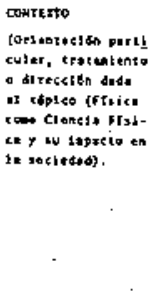 & \multicolumn{2}{|c|}{ 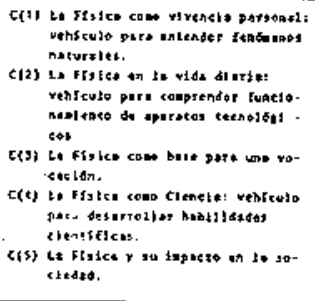 } \\
\hline 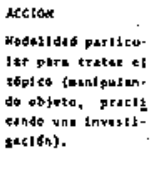 & 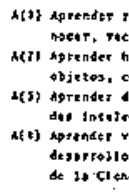 & 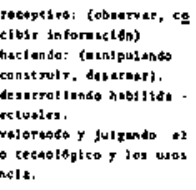 \\
\hline
\end{tabular}


Ei cuestionario de interés consta de cuatro secciones.

SECCION 1: Datos Personales, en Ia cual se solicita una información general breve acerca del alumno.

SECCXÓN 2: Tópicos, en la cual los 8 topicos se combinan sistemáticamente con contextos y accion, dando origen a 11 items por tópico.

La estructura de combinacion entre contexto y acción fue similar para todos los tópicos, de modo que las respuestas de los estudiantes puedan ser evaluadas con referencia a Tópicos, Contexto y Acción.

SECCION 3: Intereses y Tratamiento, a su vez ésta consta de tres partes. En la parte A se hace un paralelo entre interés por un determinado tópico y profundidad con que éste ha sido tratado, si el alumno lo ha estudiado. En la parte B se busca relacionar interés por un tema de Física, tratado en determinada forma, y frecuencia con que dicha forma de tratamiento ha sido utilizada en clase (contex to). En la parte $C$ se busca relacionar interés y posibles acciones a realizar en una clase de Física, y frecuencia con que dicha actividad ha sido realizada en clase (Acción).

SECCION 4: El colegio y su entomo; esta parte del cuestionario pretende identificar las variables prioritarias fuera y dentro del colegio, que podrían influir en el interés del alumno, tales como: percepciones del alumno frente a determinadas situaciones, la actuación de los padres respecto de Física y Tecnología, la utilización del tiempo libre relacionado con Física, la metodología usada en las clases de Física o Ciencias Naturales, la opinión del alumno respecto a sí mis mo frente al aprendizaje de Física.

\section{Comentario}

La versión en español del cuestionario de intereses abre insospechadas posibilidades para el mejoramiento del proceso Enseñanza Aprendizaje de la asignatura de Física en nuestro país, pues, a partir de los resultados de su aplicación a nivel nacionak, permitirá tomar conciencia, con bases concretas de la necesidad de cambios de estrategias de Enseñanza-Aprendizaje a nivel de aula; y lo que es aún más importante, la real posibilidad de tomar decisiones sobre posibles cambios, considerando la opinión del alumno. El análisis de los resultados permitirá además identificar las estructuras de intereses en relación a Física y Tecnología a través de los años de escolaridad que va cursando el alumno, como también poder identificar aquellas variables que inhiben o estimulan el desarrollo de los intereses en esta área.
Una vez que se tengan resultados concre. tos del trabajo de Investigación, se darán a conocer mediante artículos de avance en esta misma publicación.

NOTA: Como el cuestionario es un material muy valioso y dada su extensión no puede publicarse en la revista, todo aquél que desee una copia del mismo, debe enviar a la profesora María Calderón Brito la suma de U\$ 5 , para costear fotocopiado y gastos de correo aéreo.

\section{LAS REPRESENTACIONES SIM- BÓLICAS EN LA EVALUACIÓN DEL CONOCIMIENTO EN FÍSICA}

\section{F. Javier Perales Palacios.}

Departamento de Didactica de las Ciencias Experimentales. Escuela Universitaria de Magisterio. Granada.

En la Sección de esta revista "Secciones Bibliográficas Temáticas" aparecerá la revisión efectuada por nosotros sobre "Las representaciones simbólicas del conocimiento y su aplicación en la Enseñanza de las Ciencias", donde hemos tratado de contextualizar este tópico psicológica y didácticamente. Por otro lado, en la presente sección pretendemos dar cuenta de algunos resultados -simplemente enunciados allí- obtenidos de la utilización de los mapas conceptuales como técrica de evaluación, así como de su cuantificación y correlación con variables educativas relevantes.

La muestra participante en la experiencia correspondía a un grupo de estudiantes de segundo curso de Magisterio y las unidades temáticas del programa de Física General, objeto de evaluación, Cálculo Vectorial y Óptica Geométrica. Una vez concluida la exposición del tema, solicitamos de los alumnos la entrega voIuntaria de los organigramas de dichos temas. Debe señalarse que ios alumnos conocían este instrumento de representación simbolica a través de su inclusión en una breve programación de cada tema del cuestionario que se les entregaba a su inicio.

El análisis efectuado en los mapas conceptuales conlleva dos fases, una cualitativa donde se diagnostican, tanto las omisiones en los conceptos representados y relaciones entre ellos, como los errores conceptuales, es decir, consideración de relaciones no pertinentes, no discriminación conceptual o alteración de la secuenciación temporal de los conceptos, siempre en contraste con mapas conceptuales "ideales" đe los tópicos impartidos. En segundo lugar, la fase cuantitativa incluye la asignación a cada individuo de puntuaciones de omisión, de error y la suma de éstas.

Los resultados de la primera fase mostraron, en ambas unidades temáticas, una mayor incidencia de la frecuencia de omisiones que la de errores conceptuales. Así, por ejemplo, en Cálculo Vectorial, un $88 \%$ de los alumnos no relacionaron la suma de vectores con la diferencia, o un $78 \%$ no consideraron el momento de un vector respecto de un punto, como un caso particular del producto vectorial de dos vectores... Para el tema de Óptica Geométrica, un $100 \%$ de los alumnos no incluyeron la asociación de lentes o un $67 \%$ no dedujeron el dioptrio esférico a partir de un sistema óptico estigmático...

En cuanto al procedimiento correlacional, éste permití contrastar la significación de los coeficientes de correlación de Pearson entre las puntuaciones individuales mencionadas antes $\mathrm{y}$ diversas variables del ámbito cognitivo, académico y social (Perales et al. 1989b). Los datos obtenidos muestran que, para la puntuación resultante de sumar las omisiones y errores conceptuales en las dos unidades temáticas consideradas, se produce una correlación significativa y negativa de otros estudios universitarios", "calificactón en Selectividad" y "profesion de la madre".

Como tuvimos ocasión de comentar en nuestro trabajo mencionado líneas artiba (Perales. 1989a), la progresiva aplicación de técnicas como las descritas en el mismo contribuixán, a nuestro juicio, a clarificar aspectos importantes de la estructura de las disciplinas cientfficas y de su comprensión por parte del alumnado.

\section{Bibliografia}

Perales, F.J., 1989a. Las representaciones simbolicas del conocimiento y su aplicación en la Enseñanza de las Ciencias, Enseñanza de las Ciencias (en prensa).

Perales, F.J., Nievas, F. y Cervantes, A., $1989 \mathrm{~b}$. Misconceptions on geometric optics and their relation with relevant educative variables, International Journal of Science Education (en prensa). 
LA ENSEÑANZA DE LAS BIOTECNOLOGIAS

Adela Figueroa Panisse

Revista "O Ensino". Apartado 266. Pontevedra. Apartado 1036 Braga. Portugal.

El sistema de enseñanza puede ser un instrumento de pervivencia de viejas estructuras sociales, o, como muchos enseñantes lo consideran hoy por hoy, puede ser concebido como agente de cambio social, como un sistema de inserción social de individuos formados profesional y socialmente para una sociedad democrática en profundo cambio.

La revolución en el campo de la biología determina nuevas concepciones en la relacion social y familiar, pues debido a Ios avances en este campo el hombre puede hoy controlar su vida como individuo y como especie. La capacidad del hombre actual de manipular la materia viva debe ser encarada en las aulas desde edades relativamente tempranas conforme a la capacidad de comprensión de los estudiantes. En el ámbito de la enseñanza secundaria ese tema puede ser tratado en el conjunto de las materias de Ciencias Biológicas.

Dada la reforma del sistema educativo que el MEC se propone llevar a cabo, estas nuevas concepciones biologicas deben ser encuadradas en la nueva estructura de la enseñanza secundaria.

En aquella parte denominada "tronco común" $\left(7^{\circ}, 8^{\star}, 9^{2}\right.$ y $\left.10^{\circ}\right)$ los avances de las biotecnologías pueden ser incluidas dentro de las programaciones generales del área de Ciencias Físico-Biológicas.

En la parte del Bachillerato Superior o profesionalizante, la posibilidad de introducir materias optativas da una opor* tunidad más relevante al campo específico de las Biotecnologías muchas de cuyas técnicas pueden ser desarrolladas en los centros de enseñanza secundaria con vistas a la profesionalización, y también como introducción a futuros estudios más especializados teniendo en óptica el gran desarrollo que las industrias $\mathrm{I}+\mathrm{D}$ tendrán, dentro de la política general de las Comunidades Europeas.

Por eso es fundamental la actualización del profesorado tanto teórica como práctica para dar via de salida a una población juvenil que se enfrenta a una socie- 